\title{
Greedy Face Routing with Face ID Support in Wireless Networks
}

\author{
Shao Tao \\ School of Computing \\ National University of Singapore \\ Email: shaot@comp.nus.edu.sg
}

\author{
A. L. Ananda \\ School of Computing \\ National University of Singapore \\ Email: ananda@comp.nus.edu.sg
}

\author{
Chan Mun Choon \\ School of Computing \\ National University of Singapore \\ Email: chanmc@comp.nus.edu.sg
}

\begin{abstract}
Geographic face routing provides an attractive way for packet delivery in wireless networks due to its high reliability and low overhead. A good face routing protocol should provide guaranteed packet delivery and efficient routing paths. In this paper, we present a new face routing method named GFRIS that has both features by actively probing each face for the face size and the unique face identification sequence - face ID. Face switch occurs only if the outgoing edge intersects with the local minimum-destination line at a progressing location and the edge is shared between two different faces. To avoid the huge performance penalty when selecting an inefficient face traversal direction on a large face, GFRIS uses the face size to trigger the bounded face traversal procedure as proposed earlier in GOAFR+. Simulation results show that, by using face ID to assist face switch and adaptively applying the normal and bounded face traversal rules according to the face size, GFRIS achieves lower path stretch factor compared to GFG, GPSR, GFG2 and GOAFR+. The worst case performance of GFRIS is even better than that of GOAFR+ in critical node densities from 4 to 7.

Keywords - geographic routing, face identification, face switch, guaranteed delivery, path stretch factor
\end{abstract}

\section{INTRODUCTION}

The traditional wireless ad hoc routing protocols DSR and AODV use a flooding technique to find a path from the source node to the destination, which introduces an extensive overhead on a large scale network. Geographic routing protocols solve this problem by making routing decisions in a distributed manner, based on coordinates retrieved through GPS devices or localization methods[1]. Each wireless node is aware of the location of its neighbors and the destination. The next hop is computed from the location information, such that the distance metric is minimized, in terms of the angle towards the destination[2][3], the Euclidean[4] or projected[5] distance. Since greedy forwarding method will fail on local minimum, the face routing method[6] was proposed as a recovery measure to enhance packet delivery ratio for geographic routing.

Although face routing can improve delivery ratio, without identifying the faces, the face switch procedure may pass the packet to a wrong face and cause delivery failures[7]. As the efficient face traversal direction is unpredictable, face traversal by simply following the right hand rule has an unbounded worst case performance. The bounded search algorithm[8] can protect the worst case performance; however, applying bounded search universally will cause redundant link traversal on small faces and degrade the average case performance.
To alleviate the above two problems, we present a new face routing protocol named Greedy Face Routing with Identification Support(GFRIS). The GFRIS protocol probes each face efficiently for a unique identification sequence and measures the face size. The face switch takes place if the target region belongs to a new face closer to the destination. The GFRIS protocol combines both normal face traversal and bounded face traversal techniques. The bounded face traversal is applied on large faces where selecting the wrong direction may generate longer paths and the normal face traversal is used otherwise. By using face ID to assist face switch and applying both normal/bounded face traversal techniques adaptively, the GFRIS protocol can obtain the smallest path stretch value in average case and worst case scenarios.

The rest of the paper is organized as the following. The related work on geographic face routing and planarization techniques will be given in Section II. In Section III, we will describe the details of the face probing, face routing and bounded face traversal procedures in GFRIS. The performance comparison between GFRIS and the previous protocols GFG[6], GPSR [9], GOAFR+[8] and GFG2[7] will be given in Section IV. Finally, the conclusion and future works will be presented in Section V.

\section{RELATED WORK}

The GFG[6] protocol is one of the earliest protocols that combine the face routing and greedy forwarding techniques to achieve guaranteed packet delivery and efficient routing paths. The similar idea was also used in the GPSR[9] which applied the face routing method in mobile ad hoc networks. As both GFG and GPSR use the simple right/left hand rule to traverse the faces, the packets may travel on a longer side of face before the next face switch, even if a much shorter path exists in the opposite direction. The GOAFR+[8] protocol was proposed to improve this worst case performance by doing a bounded search within each face. The bounded search procedure visits the face in both directions to find a node closer to the destination. The optimal worst case performance is $O\left(c^{2}\right)$, where $c$ refers to the shortest path cost in terms of hop count or distance. The GPVFR[10] protocol enables each node to store information for multi-hop neighbors on the same face such that the nodes have more candidates to search for a next hop during the greedy forwarding stage. The face 
routing method can be further improved by using the shortcut paths[11] in high node density regions. A detailed survey on the geographic face routing protocols is presented in [12].

The face routing algorithms require a planar topology created from the full connectivity graph, such that no two links intersect with each other. The commonly used planar graphs include the Relative Neighbor Graph(RNG)[13] and the Gabriel Graph(GG)[14], both of which can be constructed in a distributed manner efficiently. The Localized Delaunay Triangulation Graph(LDT)[15] is a $t$-spanner of the original connectivity graph. The shortest path that can be found in a LDT graph for a node pair is no more than a constant $t$ times longer than the shortest path in the original graph. The RNG and GG planarization techniques require the unit disk assumption, which is invalid in a real network deployment due to asymmetric links, interference or obstacles. The Cross Link Detection Protocol(CLDP)[16] probes the crossing links before removal, thus it can avoid the network partition problem and generate a practical topology graph for face routing.

Recently, Kim et al[16] showed that some static face switch and face traversal rules may actually fail to deliver packets on a connected planar graph. Ivan Stojeminović et al[7] did a further exploration on this issue and proposed a new face switch algorithm that guarantees packet delivery on all planar graphs. In this paper, we call the face routing algorithm in [7] "GFG2". Our face routing protocol GFRIS differs from GFG, GPSR, GOAFR+ and GFG2, as GFRIS uses active probing to identify each face and provides enhanced face switch through the face identification sequence. Unlike previous face routing methods, where either normal face traversal or bounded face traversal is applied, GFRIS employs both methods and adjusts the face traversal strategy based on the face size and the distance to destination, in order to obtain a balanced performance for both average case and worst case topology scenarios.

\section{Protocol Design}

The GFRIS protocol probes each face for a unique ID to guide the face switch during normal face traversal. The selective bounded face traversal procedure is activated once the greedy forwarding fails on large faces. In this section, we will introduce the face probe, face switch and bounded face traversal methods used by the GFRIS protocol.

\section{A. Face ID Probe}

The void area enclosed by a series of links connected head to tail is named a "face". As shown in Fig. 1, a node $N$, with only location of all its one hop neighbors, will not know if its neighbors are incident to different faces(Fig. 1(a)) or the same face(Fig. 1(b)). The face probe algorithm sends probe packets along the links to obtain a unique face identification sequence. We assume the clock in each wireless node is synchronized and each node has a unique identification number.

Before presenting the details of face probing procedure, two terms need to be defined: angle and faceid. An angle is the area bounded between two neighboring links around a node $N$. As given in Table. I, the angle structure contains three

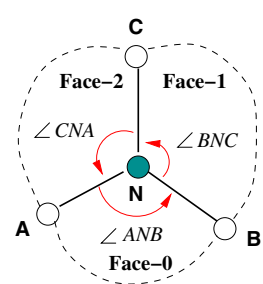

(a) 3 angles in 3 faces

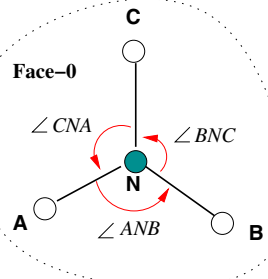

(b) 3 angles in 1 face
Fig. 1. Node $N$ with 3 Neighbors $A, B$ and $C$

parts: $c c w_{-}$start, $c c w_{-}$end and faceid. ccw_start is the first neighboring node on the angle that can be reached by turning a line in counter clock wise direction around node N. $c c w \_$end is the second neighboring node in the angle. faceid is the identification sequence of the face that the angle belongs to. In Fig. 1(a), node $N$ has three angles $\angle A N B, \angle B N C$ and $\angle C N A$. For angle $\angle A N B$, the $c c w_{-}$start is $A, c c w_{-} e n d$ is $B$ and the faceid is the identification sequence of Face-O.

TABLE I

Format of AN ANGLE STRUCTURE

\begin{tabular}{|r|c|l|}
\hline Field & Type & Meaning \\
\hline \hline$c c w \_s t a r t$ & int & node on the initial link of the angle \\
\hline$c c w \_e n d$ & int & node counter-clock wise to $c c w \_s t a r t$ \\
\hline face_id & Face ID & ID of the face containing the angle \\
\hline
\end{tabular}

The components of a faceid structure are listed in Table. II. The timestamp field contains the time when a face probe packet was sent. The prober_id field contains the node number of the sender for the probe. The seqno field is the sequence number that is incremented by one after sending each face probe packet. The face probe is triggered when a new neighboring node or a link failure is detected. Each face probe packet will carry these three fields and traverse the face boundaries by left hand rule.

TABLE II

FORMAT OF A FACE ID STRUCTURE

\begin{tabular}{|r|c|l|}
\hline Field & Type & Meaning \\
\hline \hline timestamp & double & time when a face probe is sent \\
\hline prober_id & int & identify of the node probing the face \\
\hline seqno & int & sequence number of the face probe \\
\hline
\end{tabular}

Let H.t be the timestamp, H.p be the prober_id and H.s be the seqno field of a face ID structure $H$. For two face IDs $H_{1}$ and $H_{2}$, we declare that face ID $H_{2}$ is newer than face ID $H_{1}$ (or $H_{1}$ is older than $H_{2}$ ), if one of the following conditions holds:

- $H_{2} . t>H_{1} . t$

- $H_{2} \cdot t=H_{1} \cdot t$ and $H_{2} \cdot p>H_{1} \cdot p$

- $H_{2} . t=H_{1} . t$ and $H_{2} . p=H_{1} . p$ and $H_{2} . s>H_{1} . s$

When a face probe packet $p$ carrying a face ID value $p . H$ arrives at a node $N$ from angle $\alpha$ with face ID $\alpha . H$, if $p . H$ 
is newer than $\alpha . H$, node $N$ will update the face ID record in angle $\alpha$ such that $\alpha . H=p . H$. If the face ID in packet $p . H$ is older than the face ID $\alpha . H$ stored in the intermediate node $N$, packet $p$ is considered as a stale probe packet. A face probe packet will be removed from the network if it comes back to its sender or an intermediate node finds that the probe packet is stale. The face ID competence rules are demonstrated in Fig. 2. The start up time for each face probe is delayed by a random amount of time in order to avoid simultaneous face probes, in case a new node or a link failure is detected by multiple nodes. If node $N$ has a scheduled face probe packet $p^{\prime}$ whose face id $p^{\prime} . H$ is older than $p . H$, the probe packet $p^{\prime}$ with stale face ID information will be cancelled.

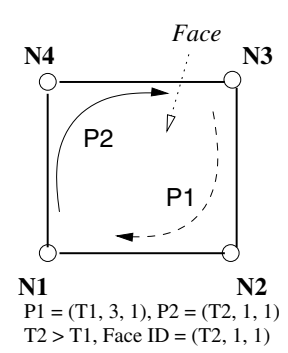

(a) Timestamp

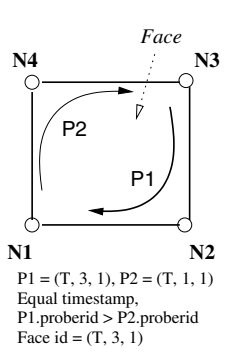

(b) Prober ID

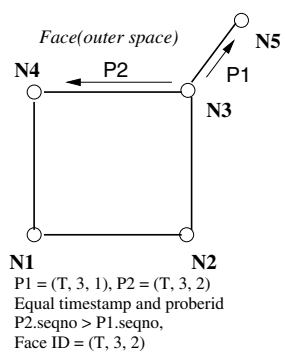

(c) Seq No.
Fig. 2. Face ID Update Rules

The probe packet with the most recent face ID information will traverse the complete face and update the angle record at each node along the way. Once the face probe procedure is completed, each node will maintain a list of angles with the corresponding face ID value for the face routing procedure.

\section{B. Face Routing with ID Support}

According to the face routing classification measures defined in [7], GFRIS uses both left and right hand rules for face traversal. The face switch occurs at the crossing link, if the face IDs for both regions on two sides of the intersecting link are different. The after crossing variant is used during face switch. Assume a packet $p$ arrives at node $N$ from the previous node $P$. Node $N$ has a link $N K$ intersecting with line $\overline{S D}$ at location $x$ closer to destination $D . S$ is the source node or the current local minimum node where the face routing was initiated. The packet is travelling the links by dir rule, where dir is either left or right hand rule. The face switch procedure at node $N$ follows Algorithm. 1 .

A GFRIS face switch example is displayed in Fig. 3. Node $N$ has 4 neighboring nodes $A, B, C$ and $P$, which constitute 4 angles: $\angle B N A, \angle A N P, \angle P N C$ and $\angle C N B$. We assume the face IDs for these angles are $F_{1}, F_{2}, F_{3}$ and $F_{4}$ respectively. Node $N$ receives a packet $p$ from node $P$. If the face traversal direction for packet $p$ is dir $=$ left, the incoming angle for packet $p$ is $\angle P N C$, whose face ID is $F_{2}$. Otherwise, the incoming angle is $\angle A N P$ with face ID $F_{3}$. Once the incoming face ID for the packet $p$ is determined, the outgoing face ID can be retrieved by computing the outgoing angle for $p$ according to the coordinates of destination $D$ and neighboring

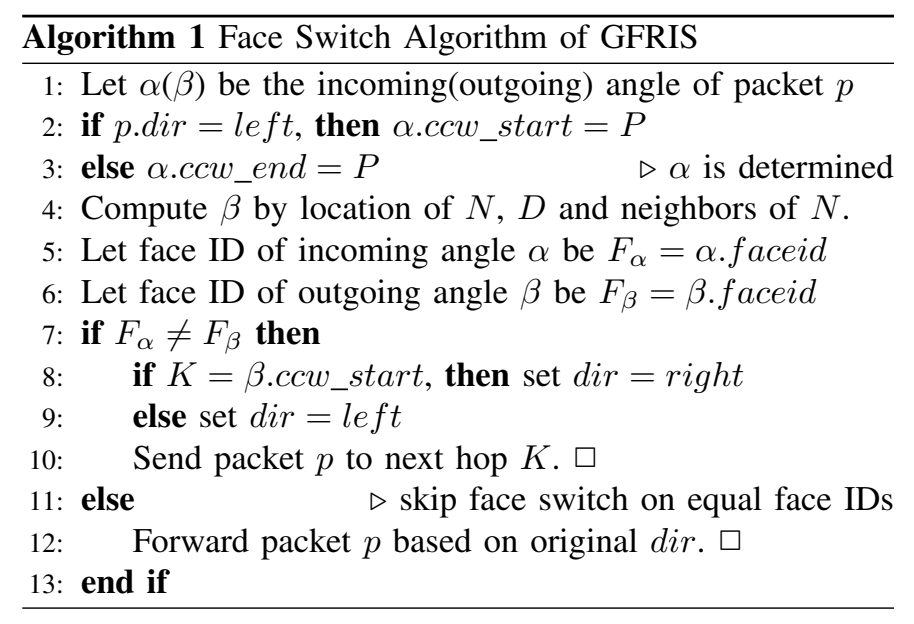

nodes of $N$. Assuming that the destination $D$ is located within the area bounded by $\angle C N B$, the outgoing angle for packet $p$ is $\angle C N B$ with face ID $F_{4}$. Thus, the incoming face ID for packet $p$ is $F_{3}\left(F_{2}\right)$ for left (right) hand rule. The outgoing face ID for packet $p$ is $F_{4}$. Since GFRIS follows the after crossing variant, the outgoing link will be the intersecting link. The face traversal direction after the switch will depend on the intersecting link. In Fig. 3, if line $\overline{S D}$ intersects with the $c c w \_s t a r t$ link $N C$ of outgoing angle $\angle C N B$, the next hop will be $C$ and the face traversal direction will be right hand rule. If line $\overline{S D}$ intersects with the $c c w \_$end link $N B$ of angle $\angle C N B$, the next hop will be $B$ and the face traversal direction will be left hand rule.

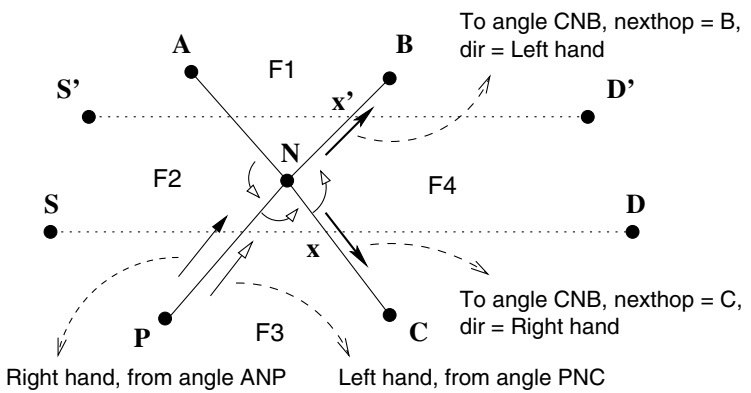

Fig. 3. Face Switch Rules in GFRIS

\section{Selective Bounded Face Traversal}

The normal face traversal technique used by GFG, GPSR cannot guarantee the worst case performance. The bounded face traversal algorithm in GOAFR+ resolves this problem by using a bounding area to find a face switch location. The drawback of applying the bounded search technique universally in all faces is that traversing small faces in alternating directions may be more expensive than traversing the face in a single direction. Thus, GFRIS employs a selective bounded face traversal method to apply bounded search to large faces only.

The face size is measured in terms of hop count during the face probe stage. While the probe packet travels along the face 
boundaries, the number of visited nodes is counted. Once a successful face probe returns to the prober, the prober node will pass the same packet for the second time to inform each node the face size. The decision about whether to use normal face traversal or bounded face traversal is made at the local minimum nodes. Assuming the local minimum node is $N$ and the destination is $D$. The term estimated ring refers to the virtual circle using $\overline{N D}$ as the diameter. The size $\varepsilon$ of the estimated ring is measured by $\varepsilon=\frac{\pi|N D|}{d_{n}}$, where $d_{n}$ is the average distance between $N$ and its neighbors. The size of face $F$ that partially contains $\overline{N D}$ is denoted as $\delta$. A face is considered as large, if the ratio $\omega$ between face size $\delta$ and estimated ring size $\varepsilon$ has $\omega=\frac{\delta}{\varepsilon} \geq 10$. As shown in Fig. 4, the search area is a circle using $|N D|$ as radius centered at $O$. The face traversal is applied within the area in alternating directions. When both directions fail to find a node where greedy forwarding can be resumed or a face switch can be initiated, the radius of the bounding circle will be doubled, $r^{\prime}=2 r$.

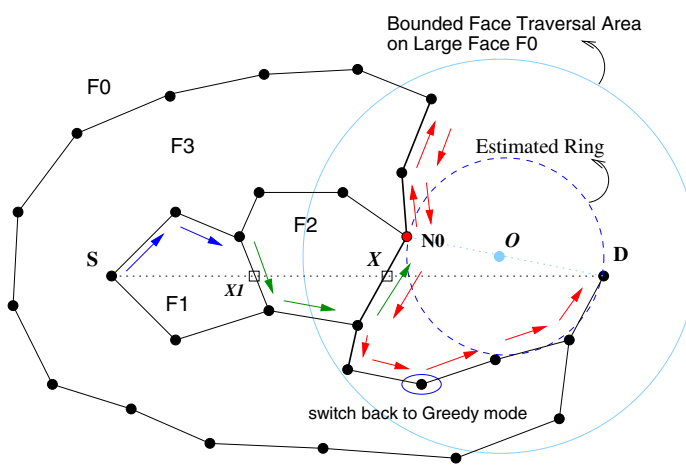

Fig. 4. GFRIS applies Bounded Face Traversal on Large Faces

\section{Performance Evaluation}

To compare the performance of GFRIS and some existing face routing protocols, we implemented greedy forwarding(Greedy), GFG, GPSR, GOAFR+, GFG2 and GFRIS in the network simulator $n s-2$. The configuration of GOAFR+ follows the original paper[8], where $\rho_{0}=1.4, \rho=\sqrt{2}$ and $\sigma=\frac{1}{100}$. Based on [7], the after crossing variant of GFG2 is used during the performance evaluation. For GFRIS, a face is considered as a large face, if the ratio $\omega$ between the face size and the estimated ring size has $\omega \geq 10$.

The configuration of simulation parameters are displayed in Table. III. The simulation area is a square with a side length of $L=400 \mathrm{~m}$. The number of wireless nodes $N$ varies from 100 to 800 with an increment size of $50(100 \leq N \leq 500)$ or $100(500 \leq N \leq 800)$. The MAC layer protocol in use is IEEE 802.11 with $11 \mathrm{Mbps}$ bandwidth and the communication range $R$ for all wireless nodes is set to $30 \mathrm{~m}$. Based on the node number, simulation area size and communication range, the 12 node densities $d=\frac{\pi R^{2}}{L^{2}} N$ covers a range from 1.77 to 14.14. For each node density, 10 random topologies are generated where the wireless nodes are placed on the simulation area
TABLE III

INPUT OF SiMULATION PARAMETERS

\begin{tabular}{|r|l|}
\hline Name & Value \\
\hline \hline Field Size & $400 \times 400 \mathrm{~m}$ \\
\hline Node Number & $100 \sim 800$ step=50(100-500), 100(500-800) \\
\hline MAC Protocol & IEEE $802.11,11 \mathrm{Mbps}$ \\
\hline Radio Range & $30 \mathrm{~m}$, CS Range $=84 \mathrm{~m}$ \\
\hline Topology & 10 topos/density, uniform location \\
\hline Connection & 100 random pairs/topology \\
\hline Routing Protocols & Greedy, GFG, GPSR, GOAFR+, GFG2, GFRIS \\
\hline Planar Graph & Gabriel Graph \\
\hline
\end{tabular}

according to the uniform distribution. From each topology, 100 random node pairs are selected to be the source and the destination. The underlying planarization method used in the simulation is Gabriel Graph. Out of the 12000 node pairs, 8202 connections are valid. We let the six routing protocols to route 3 packets for each connection and use the packet delivery ratio and average path stretch factor as the performance metrics. The initial cost of finding the face IDs is negligible when applying the preemptive face probing scheme on a static wireless network.

\section{A. Packet Delivery Ratio}

The packet delivery ratios of the six protocols are shown in Fig. 5. The Greedy protocol has a "V" shaped curve, which indicates the fact that at the critical density region $4 \sim 7$, the pure greedy forwarding method will frequently encounter local minimum cases and fail to delivery packets even if a path exists. The lowest delivery rate for Greedy occurs at density of 5.3, where only $32.6 \%$ of the packets are delivered. All the geographic face routing protocols exhibit high reliability across the entire node density region. The packet delivery ratios for GFG, GPSR, GOAFR+, GFG2 and GFRIS remain between $98.9 \%$ to $100 \%$.

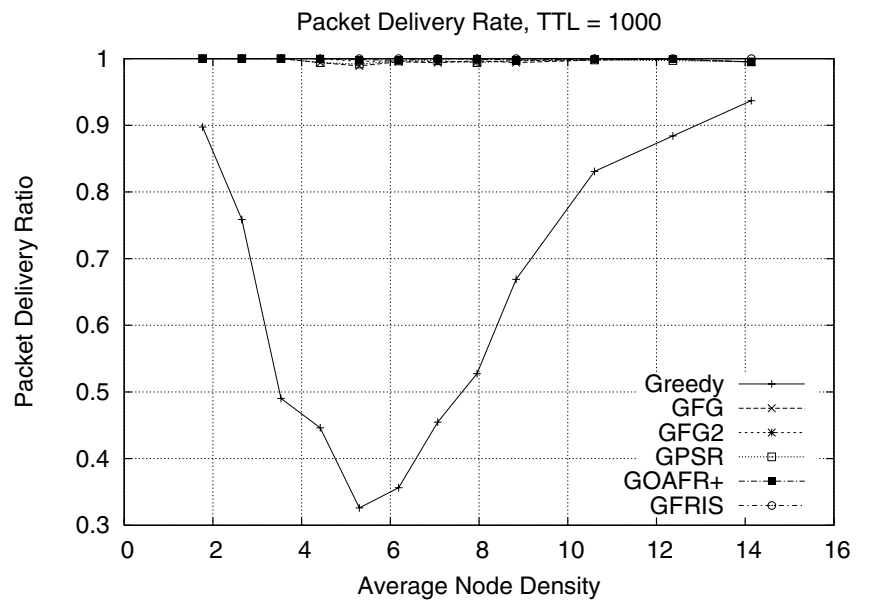

Fig. 5. Packet Delivery Ratio of Six Protocols

The detailed packet delivery failures are listed in Table. IV. Total.F is the total number of failures. Proto.F is the number 


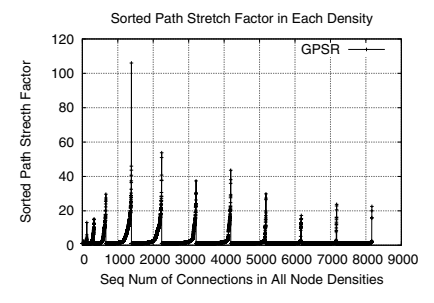

(a) GPSR

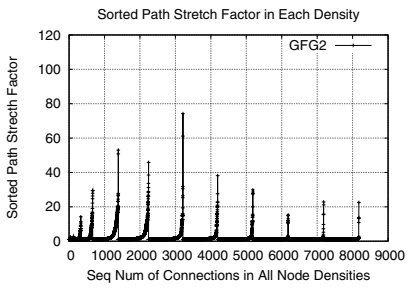

(b) GFG2

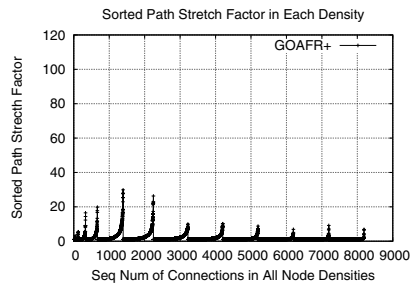

(c) GOAFR+

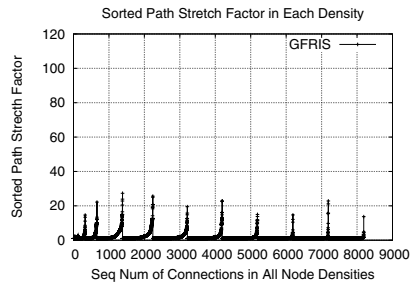

(d) GFRIS

Fig. 6. Sorted Path Stretch Factors at Each Node Density: long tails in each density are the worst case performance

of failures caused by the routing protocol. IFQ.F and MAC.F are the routing failures in the MAC layer when ARP resolution fails or MAC collision occurs. Out of the 8202 connected node pairs, the Greedy protocol failed $36.9 \%$ of them, most of which are caused by the protocol layer. None of the face routing protocols encounter any protocol level failure, despite the fact that some of them may fail in Gabriel Graphs[7][16]. Although the MAC failures in $n s-2$ might occur in random patterns, packets travelling longer routing paths do have higher possibility of getting dropped due to MAC layer contention. The GFG, GPSR, GOAFR+ and GFG2 protocols have a small number of routing failures caused by the MAC layer, while GFRIS has not encountered any.

TABLE IV

Packet Delivery FaILURes at Various LeVels

\begin{tabular}{|c|c|c|c|c|}
\hline Protocols & Total.F & Proto.F & IFQ.F & MAC.F \\
\hline \hline Greedy & 3030 & 3019 & 3 & 8 \\
\hline GFG & 37 & 0 & 4 & 33 \\
\hline GPSR & 33 & 0 & 4 & 29 \\
\hline GOAFR+ & 13 & 0 & 4 & 9 \\
\hline GFG2 & 26 & 0 & 4 & 22 \\
\hline GFRIS & 0 & 0 & 0 & 0 \\
\hline
\end{tabular}

\section{B. Path Stretch Factor}

The path stretch factor is a critical metric to evaluate the efficiency of face routing algorithms. Given a shortest path of length $H_{s p}$, a face routing method that generates a routing path of length $H_{\text {proto }}$ has a path stretch value of $\lambda_{i}=\frac{H_{\text {proto }}}{H_{s p}}$. The average path stretch factors is calculated as $\lambda=\left(\sum_{i=1}^{n^{s p}} \lambda_{i}\right) / n$ for $n$ test cases. A routing protocol with a smaller average path stretch factor outputs shorter paths in general and achieves higher efficiency.

We compare the packet delivery paths returned by the routing protocols with the shortest ones found by the BellmanFord algorithm. Within each node density ranging from 1.77 to 14.14 , we sort the test cases according to the path stretch factor value. The sorted path stretch graph in Fig. 6 shows an increasing curve for each node density. The long tails of these curves represent the worst case performance at that particular density. GPSR and GFG2 have significantly larger path stretch values at the worst case scenarios, because the face traversal they utilize does not provide a fall-back mechanism when an inefficient path is encountered. Both GOAFR+ and GFRIS can obtain apparently improved worst case performance, with the assistance from the bounded face search strategy.

We further sort the path stretch values across the entire 12 node densities and divide them into 4 categories: best cases, average cases(first half, second half) and the worst cases, then we compute the average path stretch factor at each category. The categorized average path stretch factors are displayed in Fig. 7, which shows the performance of the face routing protocols under various topology conditions. In the best cases at Fig. 7(a), all the face routing protocols obtain a path stretch nearly equal to 1 . In the average cases at Fig. 7(b) and Fig. 7(c), the path stretch values start to increase, as greedy forwarding fails at local minimum locations and the face routing step is activated to accomplish the delivery. In the best case and average case categories, the performance of GOAFR+ is evidently degraded because it applies the same bounded face search method over all cases universally. GFG and GPSR have lower average path stretch values than GOAFR+. Both GFG2 and GFRIS achieves the best path stretch performance, as a result of their efficient face switch strategy. The worst case scenarios are illustrated in Fig. 7(d). In this category, the path stretch values for all the face routing methods increase dramatically, which makes it the dominant factor when computing the overall average path stretch performance. Without the bounded face traversal procedure, at node density of 5.3, the worst case path stretch of GFG, GPSR and GFG2 reaches 16.3, 13.36 and 9.25. By applying the bounded face search, the GOAFR+ protocol manages to maintain a lower path stretch of 7.56. The GFRIS protocol makes further improvement over GOAFR+ by selectively using the bounded face traversal method on the large faces and obtains the lowest average worst case path stretch of 6.26 , which is $17 \%$ less than GOAFR+, $32.4 \%$ less than GFG2, 53.2\% less than GPSR and 61.3\% less than GFG.

From Fig. 7, it can be seen that using merely normal face traversal will not maintain low path stretch in worst case topologies and applying merely bounded face search will degrade average case performance. The GFRIS protocol employs both methods adaptively and manages to achieve the best performance in all test case scenarios. The overall average path stretch is given in Fig. 8, where the performance of GFRIS remains the best across the entire range of node densities. 


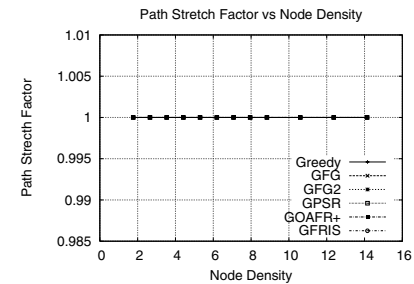

(a) $0 \% \sim 25 \%$ of Max (Best Cases)

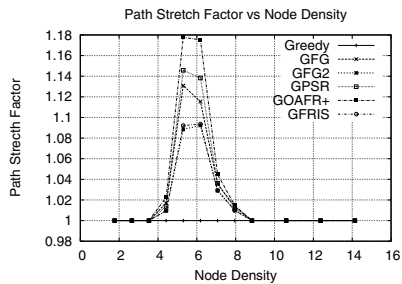

(b) $25 \% \sim 50 \%$ Cases, first half)

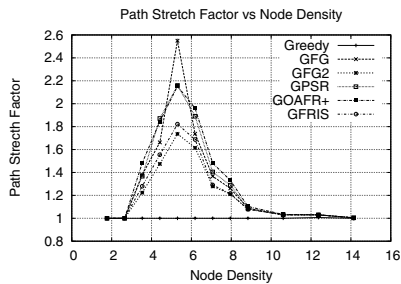

(c) $50 \% \sim 75 \%$ of $\mathrm{Max}$ Cases, second half)

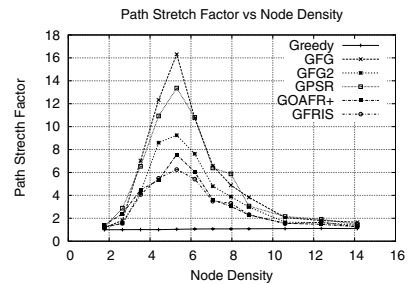

(d) $75 \% \sim 100 \%$ of Max (Worst Cases)

Fig. 7. Categorized Average Path Stretch Factors: from best cases to worst cases

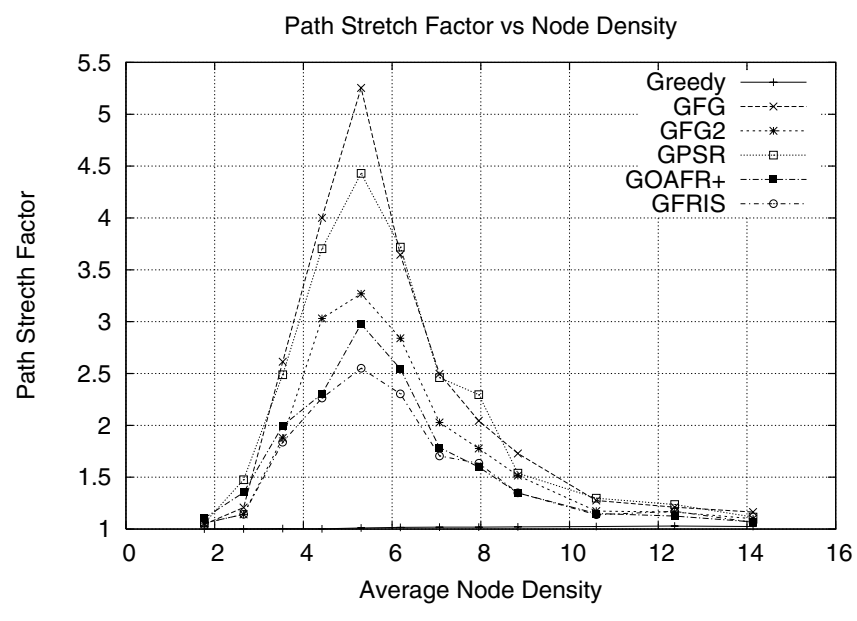

Fig. 8. Average Path Stretch Factor over All Test Cases

\section{CONClusion}

In this paper, we described a new face routing algorithm - GFRIS, which is able to identify each unique face on planar graphs and use the face ID information to initiate the face switch procedure. By utilizing face identification sequence, the GFRIS protocol can obtain efficient routing paths and reduce the face traversal oscillation. GFRIS employs normal face traversal and bounded face traversal adaptively and achieves improved performance than other face routing protocols evaluated, in terms of path stretch under both average case and worst case scenarios.

In future work, we will systematically explore the relation between bounded face search performance and the underlying topologies. We will also work on the multi-path geographic face routing algorithms, such that the data packets can be routed with higher reliability and traffic load is balanced across the network. Due to the dependence on planar topologies, the geographic face routing algorithms need to be modified to operate in a three-dimensional space, where the traditional planarization methods are no longer applicable. As the wireless node deployment is done in 3-D space by nature, geographic face or space routing in higher dimensions deserves further investigation.

\section{REFERENCES}

[1] Yao Zhao, Bo Li, Qian Zhang, Yan Chen, and Wenwu Zhu. Efficient hop id based routing for sparse ad hoc networks. In Proceedings of the 13th International Conference on Network Protocols, pages 179-190, 2005.

[2] Evangelos Kranakis, Harvinder Singh, and Jorge Urrutia. Compass routing on geometric networks. In Proceedings of the 11th Canadian Conference on Computational Geometry, pages 51-54, 1999.

[3] Stefano Basagni, Imrich Chlamtac, Violet R. Syrotiuk, and Barry A. Woodward. A distance routing effect algorithm for mobility(DREAM). In Proceedings of the 4th Annual ACM/IEEE International Conference on Mobile Computing and Networking, pages 76-84, 1998.

[4] Gregory G. Finn. Routing and addressing problems in large metropolitan-scale internetworks. Technical Report ISI/RR-87-180, University of Southern California Marina del Rey Information Science Institute, 1987.

[5] Hideaki Takagi and Leonard Kleinrock. Optimal transmission ranges for randomly distributed packet radio terminals. IEEE Transactions on Communications, 32(3):246-257, 1984.

[6] Prosenjit Bose, Pat Morin, Ivan Stojmenović, and Jorge Urrutia. Routing with guaranteed delivery in ad hoc wireless networks. Wireless Networks, 7(6):609-616, 2001.

[7] Hannes Frey and Ivan Stojmenović. On delivery guarantees of face and combined greedy-face routing in ad hoc and sensor networks. In Proceedings of the 12th International Conference on Mobile Computing and Networking, pages 390-401, 2006.

[8] Fabian Kuhn, Roger Wattenhofer, Yan Zhang, and Aaron Zollinger Geometric ad-hoc routing: of theory and practice. In Proceedings of the 22nd Annual Symposium on Principles of Distributed Computing, pages 63-72, 2003.

[9] Brad Karp and H. T. Kung. GPSR: Greedy perimeter stateless routing for wireless networks. In Proceedings of the 6th Annual International Conference Mobile Computing and Networking, pages 243-254, 2000.

[10] Ben Leong, Sayan Mitra, and Barbara Liskov. Path vector face routing: Geographic routing with loal face information. In Proceedings of the 13th IEEE International Conference on Network Protocols, pages 147$158,2005$.

[11] Susanta Datta, Ivan Stojmenović, and Jie Wu. Internal node and shortcut based routing with guaranteed delivery in wireless networks. In Proceedings of the 21st International Conference on Distributed Computing Systems Workshop, pages 461-466, 2001.

[12] Hannes Frey. Scalable geographic routing algorithms for wireless ad hoc networks. IEEE Network, 18(4):18-22, 2004.

[13] Jerzy W. Jaromczyk and Godfried T. Toussaint. Relative neighborhood graphs and their relatives. Proceedings of the IEEE, 80(9):1502-1517, 1992.

[14] K. Ruben Gabriel and Robert R. Sokal. a new statistical approach to geographic variation analysis. Systematic Zoology, 18(3):259-278, 1969.

[15] Xiang-Yang Li, Gruia Calinescu, and Peng-Jun Wan. Distributed construction of a planar spanner and routing for ad hoc wireless networks. In Proceedings of the 21st Annual Joint Conference of the IEEE Computer and Communications Societies, volume 3, pages 1268-1277, 2002.

[16] Young-Jin Kim, Ramesh Govindan, Brad Karp, and Scott Shenker. On the pitfalls of geographic face routing. In Proceedings of the 2005 Joint Workshop on Foundations of Mobile Computing, pages 34-43, 2005. 